\title{
Cervical spinal cord injury and ultrasound evaluation of diaphragm function
}

\section{Letter to Editor}

Traumatic spinal cord injury requires the collaboration of a multidisciplinary team, both for the specialized treatment of the acute phase and for secondary complications. In respiratory management in the acute phase, early evaluation of respiratory function is a priority to anticipate the need for ventilatory support. The location and degree of cervical spinal cord injury affects both the onset of mechanical ventilation and the successful weaning of the respirator. Injuries above C5 and ASIA A are among the factors with the highest risk for developing respiratory failure. It was found that a motion index below 10 and the presence of respiratory complications are also good predictors. The duration of mechanical ventilation is also determined by age (over 45 years), concomitant lung diseases, smoking, low level of consciousness (GCS less than 9), comorbidities and an Injury Severity Score less than or equal to $16 .{ }^{1}$

In $\mathrm{C} 1-\mathrm{C} 2$ injuries, there is no effective respiratory musculature. The diaphragm is innervated by the phrenic nerve, which is composed of the $\mathrm{C} 3, \mathrm{C} 4$ and $\mathrm{C} 5$ cervical nerves. Therefore, patients with complete motor injury above C5 will almost invariably need ventilatory support. ${ }^{2}$ Injuries below C5 result in paralysis of the intercostal and abdominal musculature; therefore, some patients will require respiratory support. The loss of respiratory capacity in patients with cervical spinal cord injury occurs due to exhaustion of the musculature involved in the ventilatory process, haemorrhage or ascending spinal oedema, accumulation of secretions, atelectasis, other associated traumas or other conditions of the patient. Tracheotomy is required in $11-35 \%$ of patients with cervical spinal cord injury for the treatment of pulmonary complications and pulmonary hygiene. ${ }^{3}$ Tracheotomy reduces the duration of respiratory cycles, improves lung capacity to mobilize secretions and reduces resistance of the respiratory tract. As a result, the weaning of the ventilator becomes easier and reduces the mechanical ventilation duration. There is evidence that early tracheotomy reduces the incidence of pneumonia, shortens the duration of mechanical ventilation, reduces the stay in the ICU and reduces the rates of serious complications associated with orotracheal intubation, such as tracheal stenosis. ${ }^{4}$ Tracheotomy does not appear to have deleterious effects when performed shortly after cervical fixation surgery and does not promote cross infection despite the proximity of the incisions.

Ventilatory failure in patients with cervical spinal cord injury occurs more frequently after the fourth day of the injury, which is relevant for the monitoring and consideration of maintaining the intubation after early surgical intervention, especially those from cervical surgical procedures. Different tools are available to assess respiratory muscle function. However, most of them are of limited value (chest radiograph and fluoroscopy) or are not suitable for routine clinical care monitoring (magnetic resonance imaging, computed tomography, phrenic nerve stimulation). Circulatory biomarkers for respiratory muscle injury are indeed desirable. So, the evaluation of diaphragm function is carried out through medical history, physical examination, nerve conduction studies and electromyography (EMG). Nerve
Volume II Issue 3 - 2019

\author{
Luisa María Charco-Roca,' Elena Simón- \\ Polo ${ }^{2}$ \\ 'Area Specialist Practitioner (MD), Anaesthesiology and \\ Intensive Care Service, General University Hospital of Albacete, \\ Spain \\ ${ }^{2}$ Hospital Resident (MD),Anaesthesiology and Intensive Care \\ Service, General University Hospital of Albacete, Spain
}

Correspondence: Luisa María Charco-Roca,Area Specialist Practitioner (MD), Anaesthesiology and Intensive Care Service, General University Hospital of Albacete, TesifonteGallego street number 20, $5^{\circ} \mathrm{A} .02002$, Albacete, Spain, Tel + 34636667599 , Email luisacharc@gmail.com

Received: December 20, 2019 | Published: December 24, 2019

conduction and EMG studies are uncomfortable and can sometimes cause complications such as pneumothorax. Imaging techniques can be used as an approach for the evaluation of diaphragm dysfunction. Paresis or diaphragm paralysis is usually presented as an elevation on simple chest radiography. Functional imaging with fluoroscopy is a simple and effective method to diagnose diaphragm dysfunction, being classified as paralysis, weakness or eventration. Diaphragm paralysis can be observed as an absence of orthographic excursion of the diaphragm in the respiratory cycles, usually presenting a paradoxical movement with inspiration. ${ }^{5}$

Diaphragm ultrasound provides a quick approximation of diaphragm function and offers excellent interobserver reproducibility. In critically ill patients, it can be used to diagnose dysfunction or paralysis, predict success of weaning or assess respiratory effort. ${ }^{6}$ There is no consensus on the best ultrasound assessment method. The measurement of diaphragm shortening, which is the variation of thickness during inspiration/expiration, measured in the apposition zone at the 6th-8th rib, provides a simple way to diagnose paralysis or diaphragm dysfunction both unilaterally and bilaterally. Both the diaphragm excursion and the change in muscle thickening are measured by ultrasonography to evaluate the function of the diaphragm and the possible weaning of mechanical ventilation.

We concluded that respiratory failure and pulmonary complications are the main factors that contribute to the morbidity and mortality of patients with cervical or high thoracic spinal cord injury. Ultrasound evaluation of diaphragm function allows the detection of diaphragm dysfunction in patients with cervical spinal cord injury and provides early ventilatory support in anticipation of respiratory complications. Ultrasound evaluation of diaphragm provides reliable data to establish a prognosis regarding the initial needs for ventilatory support. This evaluation of respiratory function on observing adequate diaphragm mobility can also be applied to patients in the weaning phase of mechanical ventilation. Early tracheotomy is suggested within the first 10 days and offers beneficial effects in the management 
of patients with acute spinal cord injury at the cervical level who require longterm mechanical ventilation, facilitating the weaning of the respirator and reducing the stay and complications related to intubation in the intensive care unit.

\section{Conflict of interest}

There are no conflicts of interest on behalf of the authors, nor external funding sources.

\section{References}

1. Menaker J, Kufera JA, Glaser J, et al. Admission ASIA motor score predicting the need for tracheostomy after cervical spinal cord injury. $J$ Trauma Acute Care Surg. 2013;75(4):629-634.
2. Claxton AR, Wong DT, Chung F, et al. Predictors of hospital mortality and mechanical ventilation in patients with cervical spinal cord injury. Can J Anaesth. 1998;45(2):144-149.

3. Velmahos GC, Toutouzas K, Chan L, et al. Intubation after cervical spinal cord injury: To be done selectively or routinely? Am Surg. 2003;69(10):891-894.

4. Ganuza JR, Garcia Forcada A, Gambarrutta C, et al. Effect of technique and timing of tracheostomy in patients with acute traumatic spinal cord injury undergoing mechanical ventilation. J Spinal Cord Med. 2011;34(1):76-84

5. Summerhill EM, El-Sameed YA, Glidden TJ, et al. Monitoring recovery from diaphragm paralysis with ultrasound. Chest. 2008;133(3):737-734.

6. McCool FD, Tzelepis GE. Dysfunction of the diaphragm. $N$ Engl J Med. 2012;366(10):932-942. 